\title{
Viscosity Is Not a Parameter of Postdeglutitive Pharyngeal Residue: Quantification and Analysis with Scintigraphy
}

\author{
H. C. A. Bogaardt, MSc, ${ }^{1}$ J. J. Burger, $\mathrm{PhD},{ }^{3}$ W. J. Fokkens, PhD, MD,${ }^{1}$ and R. J. Bennink, PhD, MD ${ }^{2}$ \\ ${ }^{1}$ Department of Otolaryngology, Academic Medical Center, University of Amsterdam, Amsterdam, The Netherlands; ${ }^{2}$ Department of \\ Nuclear Medicine, Academic Medical Center, University of Amsterdam, Amsterdam, The Netherlands; and ${ }^{3}$ Quest International Nederland \\ BV, Naarden, The Netherlands
}

\begin{abstract}
The aim of this study was to explore the influence of viscosity on pharyngeal residue in normal healthy volunteers. Scintigraphy was used to measure pharyngeal residue in 11 healthy volunteers after swallowing three different substances (age $=20.2$ 48.3 years). The first substance was a 10 -ml solution of tap water with $0.5 \%$ xanthan with a viscosity of $4500 \mathrm{mPa} \mathrm{s}$, comparable to a yogurt drink. The second and third substances were a $0.75 \%$ xanthan and a $1.00 \%$ xanthan solution, with viscosities of 10,500 and $21,000 \mathrm{mPa} \mathrm{s}$, comparable to low-fat yogurt and $3 \%$ fat yogurt, respectively. Tap water was used as the control substance. Mean pharyngeal residue after swallowing tap water was $2.3 \%(\mathrm{SD}=1.2)$ of the initial volume in the oral cavity. Pharyngeal residue after swallowing $0.5 \%$ xanthan solution was $1.8 \%$ $(\mathrm{SD}=0.8)$, after swallowing $0.75 \%$ xanthan solution $2.6 \%(\mathrm{SD}=2.2)$, and after swallowing $1.00 \%$ xanthan solution $2.8 \%(\mathrm{SD}=1.7)$. No significant correlation between increase of viscosity and pharyngeal residue was found. In healthy persons viscosity does not seem to be a significant parameter for pharyngeal residue for boluses with viscosities ranging from tap water to solutions having a viscosity comparable to $3 \%$ fat yogurt.
\end{abstract}

Key words: Deglutition - Physiology - Radionuclide imaging - Deglutition disorders.

The residual amount of food or beverage in the pharynx after swallowing (pharyngeal residue) is an

Correspondence to: H. C. A. Bogaardt, MSc, Department of Otolaryngology, Academic Medical Center, Meibergdreef 9, 1100 DD, Amsterdam, The Netherlands; E-mail: h.c.bogaardt@amc.uva.nl important parameter in diagnostic procedures in the treatment of swallowing disorders. In common practice of management of swallowing disorders, the amount of pharyngeal residue is determined either by videofluoroscopy or by transnasal flexible endoscopy (FEES). With the help of logistic regression, Perlman et al. [1] predicted the chance of aspiration (food or liquid that gets into the lungs) based on the amount of pharyngeal residue. An odds ratio of 1.4 for aspiration was found for patients with pharyngeal residue. In this study 330 videofluoroscopies of dysphagic patients were analyzed and the amount of pharyngeal residue was rated on a four-point scale (absent, mild, moderate, severe). The study also showed that a patient with severe pharyngeal residue has an increased risk of aspiration (odds ratio of 4.0) and underlined the importance of quantifying pharyngeal residues. Dejaeger et al. [2] analyzed videofluoroscopies of 25 healthy elderly and clearly described localization of pharyngeal residue; unfortunately, they did not attempt to quantify the amount of residue.

Few attempts have been made to quantify pharyngeal residue in normal and dysphagic populations, mainly because of the limited availability of appropriate diagnostic tools. Videofluoroscopy or FEES has been used to explore physiologic parameters of swallowing [3,4]. One of the limitations of the use of these techniques is the lack of reliable interpretation of physiologic parameters. Stoeckli et al. [5] analyzed the inter- and intrajudge reproducibility of the interpretation of videofluoroscopy and suggested that the agreement for assessing pharyngeal parameters is minimal to moderate $(\kappa=0.01-0.56)$.

Scintigraphy allows quantitative measurement by using a technetium- $99 \mathrm{~m}\left({ }^{99 \mathrm{~m}} \mathrm{Tc}\right)$-labeled colloid. ${ }^{99 \mathrm{~m}} \mathrm{Tc}$ colloid is used in routine clinical practice for 
investigating gastrointestinal motility disorders of the esophagus and stomach [6,7]. The scintigraphic technique allows quantitative assessment because the amount of radioactivity (radioactive counts) can be measured with a gamma camera. Scintigraphy in dysphagia mainly is used for detection of aspiration in young children because the radiation doses are less than those of videofluoroscopy [8]. It is used less frequently in adults, although recently it has been described as a useful and reliable diagnostic tool in adult patients with oropharyngeal dysphagia [9]. Only three studies have been performed with scintigraphy to examine pharyngeal parameters of normal swallowing physiology: Cook et al. [10] used scintigraphy to study the influence of age on swallowing efficiency. Hamlet et al. [11] reported on the differences in bolus transit times and oropharyngeal residues in healthy volunteers, while Shaw et al. [12] studied the influence of bolus volume on pharyngeal residue. All these studies examined different parameters of swallowing and found different outcomes. The aim of this study was to explore the influence of viscosity on pharyngeal residue in normal healthy volunteers, where our hypothesis was that swallowing a more viscous product would result in more pharyngeal residue in healthy volunteers.

\section{Methods}

\section{Volunteers}

Eleven healthy volunteers ( 9 female, 2 male, mean age $=29.1$ years, standard deviation $[\mathrm{SD}]=8.9$ ), recruited by public advertisement, were invited to participate. All volunteers reported no history of swallowing problems. All volunteers gave written informed consent to participate in the study, which was approved by the Medical Ethics Committee of the Academic Medical Center of the University of Amsterdam.

\section{Bolus Characteristics}

Before the examination, three different substances with three different viscosities were prepared in a research laboratory. All viscosities were measured with a viscosity meter (Brookfield LVT Viscometer, model DV-II ${ }^{+}$) after $24 \mathrm{~h}$ of storage in a refrigerator at a constant temperature of $4^{\circ} \mathrm{C}$. Measurements were performed with spindle No. 63 at a velocity of $3 \mathrm{rpm}$ and viscosities were recorded after $30 \mathrm{~s}$. The substances consisted of tap water thickened with different percentages of xanthan, a polysaccharide commonly used in the food industry to thicken fluids. The first substance was a solution of tap water with $0.5 \%$ xanthan resulting in a substance with a viscosity of $4500 \mathrm{mPa}$ s, comparable to a yogurt drink. The second and third substances were tap water with $0.75 \%$ and $1.00 \%$ xanthan, resulting in viscosities of 10,500 and $21,000 \mathrm{mPa} \mathrm{s}$, comparable to low-fat yogurt and $3 \%$ fat yogurt, respectively. As reference, tap water with a viscosity of $1 \mathrm{mPa}$ s (per definition) was used. For all solutions $200 \mathrm{ml}$ was labeled with $260 \mathrm{MBq}$ of ${ }^{99 \mathrm{~m}} \mathrm{Tc}$ colloid (Hepatate, GE Health, Eindhoven, The Netherlands) dissolved in $1 \mathrm{ml} 0.9 \%$ saline solution and prepared in such a way that for $10 \mathrm{ml}$ of each solution the radiation dose was $13 \mathrm{MBq}$. After labeling, 44 syringes (4 per volunteer) were used to collect 10 $\mathrm{ml}$ of each of the four solutions (tap water, $0.5 \%, 0.75 \%$, and $1.00 \%$ xanthan). The three xanthan solutions, stored for more than $24 \mathrm{~h}$ in a refrigerator at a stable temperature of $4^{\circ} \mathrm{C}$, were mixed with the ${ }^{99 \mathrm{~m}} \mathrm{Tc}$ colloid $1 \mathrm{~h}$ before the study.

\section{Data Collection}

For this study a gamma camera (Orbiter, Siemens Medical Systems, Germany) was fitted with a low-energy all-purpose collimator. A dynamic acquisition using 0.25 -s frames was performed for $30 \mathrm{~s}$. Data were acquired and processed on a Hermes workstation (Nuclear Diagnostics, Sweden). Volunteers were asked to sit in front of the gamma camera and hold their right cheek against the collimator. The oral cavity was placed in the middle of the field-ofview. When the volunteer was placed in the right position, $10 \mathrm{ml}$ of one of the solutions was emptied in the mouth of the volunteer with a syringe. Volunteers were instructed to hold the bolus in their mouth and swallow on command. They were specifically instructed to swallow the whole bolus in one swallow and to breath through the mouth to inhibit a second swallow. Three cobalt markers were placed on the skin of the volunteers using a pen pointer: one on the mastoid bone, one on the hyoid bone, and one underneath the thyroid cartilage. The exact placement for the marker was found by palpation of the head and neck.

First, the volunteers were asked to swallow the water with ${ }^{99 \mathrm{~m}} \mathrm{Tc}$ colloid. After recording the swallow for $30 \mathrm{~s}$, the volunteers were asked to rinse their mouth with unlabeled tap water and to swallow $200 \mathrm{ml}$ unlabeled tap water to wash away any residue from the previous recording. The second recording was done with $10 \mathrm{ml}$ of the $0.5 \%$ xanthan solution, the third with the $0.75 \%$ xanthan, and the last with the $1.00 \%$ xanthan. Between recordings the volunteers were instructed to rinse their mouth and swallow $200 \mathrm{ml}$ unlabeled tap water to clear any possible residue. Each volunteer swallowed a consistency labeled with a dose of $13 \mathrm{MBq}$ four times, for a total of $52 \mathrm{MBq}$, which is equal to a radiation dose of less than 1 milliSievert (mSv). According to the ICRP62 guidelines, this is a minor radiation dose (category IIa).

After data collection the results were stored and processed on a Hermes workstation. Based on the markers, two regions of interest (ROI) were defined: the oral cavity (ROI-1), bordered by a straight line between the hyoid marker and the mastoid marker, and the pharynx (ROI-2), bordered by a straight line between the hyoid marker and the mastoid marker and a horizontal line from the thyroid marker, as described by previous studies [10, 11]. To estimate background activity during the examinations, a third ROI with a comparable surface area to ROI- 2 was defined to the right of the field of view. Figure 1 shows the three ROIs. Collected data were transferred to a spreadsheet file (Excel, Microsoft Corp., Redmond, WA), which showed the number of counts within the specific ROI at each frame $(0.25 \mathrm{~s})$ of the total acquisition. For our analysis the average count of 8 frames $(2 \mathrm{~s})$ before swallowing was used to determine the average number of counts in the oral cavity. After swallowing the average count of $4 \mathrm{~s}$ (16 frames) was used to determine the average amount of pharyngeal residue, expressed in counts. Background activity was estimated by a measurement of 4 $\mathrm{s}$; this was subtracted from the average count in the oral cavity and the pharyngeal residue. Hereafter, the number of pharyngeal counts was divided by the number of counts in the oral cavity, thus providing a percentage of pharyngeal residue. 


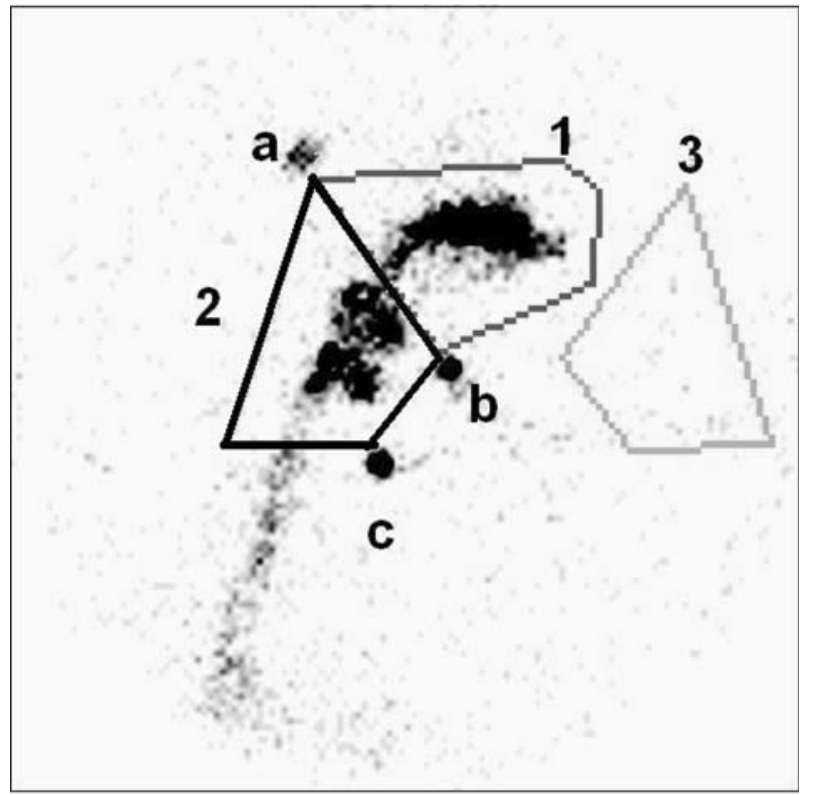

$1=$ oral cavity, $2=$ pharynx, $3=$ background radiation

$\mathrm{a}=$ mastoid marker, $\mathrm{b}=$ hyoid marker, $3=$ thyroid marker

Fig. 1. Scintigraphic evaluation with three regions of interest (ROI) $1=$ oral cavity, $2=$ pharynx, $3=$ background radiation, $a=$ mastoid marker, $\mathrm{b}=$ hyoid marker, $3=$ thyroid marker.

\section{Statistical Analysis}

The calculated percentages of pharyngeal residue were transferred to SPSS v12.0 (SPSS Inc., Chicago, IL) for further analysis. Distribution of the percentages was formally tested with the Kolmogorov-Smirnoff test for normality and was not found to be normally distributed. To explore differences in median percentages of pharyngeal residues of the three xanthan solutions to the reference (tap water) on a group level, a nonparametric (Friedman) test was used. All statistical tests were two-tailed and differences were evaluated at the $5 \%$ level of significance.

\section{Results}

The mean pharyngeal residue after swallowing tap water was $2.3 \%(\mathrm{SD}=1.2$; range $=1.1 \%-4.5 \%)$ of the initial volume in the oral cavity. The percentage of pharyngeal residue after swallowing the $0.5 \%$ xanthan solution was $1.8 \%(\mathrm{SD}=0.8$; range $=0.9 \%-2.8 \%)$, after swallowing the $0.75 \%$ xanthan solution the residue was $2.6 \%(\mathrm{SD}=2.2$; range $=0.7 \%-8.1 \%)$, and after swallowing the $1.00 \%$ xanthan solution the pharyngeal residue was $2.8 \% \quad(\mathrm{SD}=1.7$; range $=1.2 \%-6.4 \%$ )

Median percentages of pharyngeal residue were analyzed. Figure 2 shows by boxplot the distribution of residue for the four different viscosities in the 11 nondysphagic volunteers. No significant difference in the amount of residue between the four groups was found $\left(\chi^{2}=0.927, \mathrm{df}=3, p=0.819\right)$.

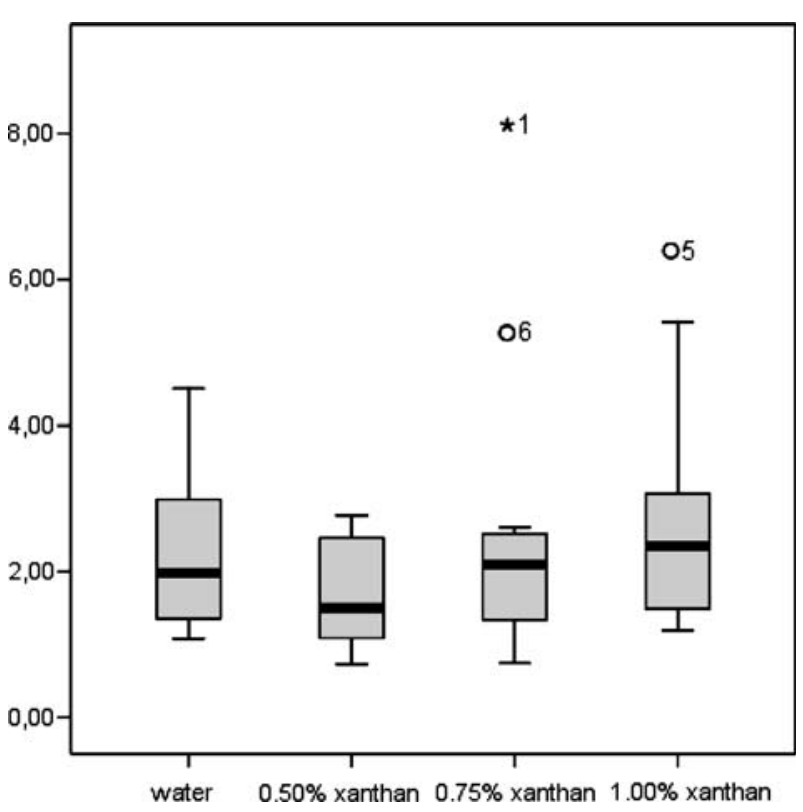

Fig. 2. Distribution of percentages residue on group level.

Although the sample size was limited, post hoc analysis showed that this study had a power of 0.96 ( $n=11$ with an effect size of 0.47 based on a SD and correlation between measurements occasions of 1.1 and 0.7, respectively). Based on these results, a power calculation showed that in future research (with a power of 0.80 and alpha of 0.05 ) at least 137 healthy volunteers would be needed to detect any possible difference in the amount of pharyngeal residue between the four groups (tap water, $0.5 \%, 0.75 \%$, and $1.00 \%$ xanthan) (Table 1$)$.

\section{Discussion}

Our study attempted to investigate the correlations between viscosity and pharyngeal residue in healthy volunteers; we did not find evidence for a positive correlation between an increase of viscosity and an increase in pharyngeal residue.

Because Shaw et al. [12] established that bolus volume does not influence pharyngeal residue, allowing extrapolation of our findings to other volumes, only one bolus volume was used in this study. It might be suggested that our volunteers drank 200 $\mathrm{ml}$ tap water after the first labeled bolus and thus the mucosa of the pharynx was relatively dry before the first estimation of pharyngeal residue. Because the larger proportion of our volunteers had drunk coffee, tea, or water in the last hour before the study, differences in dryness of the pharyngeal mucosa is not considered a confounder. 
Table 1. Pharyngeal residue in percentages with $10 \mathrm{ml}$ boluses

\begin{tabular}{lllll}
\hline No. & Water & $\begin{array}{l}0.5 \% \\
\text { Xanthan }\end{array}$ & $\begin{array}{l}0.75 \% \\
\text { Xanthan }\end{array}$ & $\begin{array}{l}1.00 \% \\
\text { Xanthan }\end{array}$ \\
\hline 1 & 1.1 & 1.2 & 0.7 & 1.2 \\
2 & 1.8 & 1.2 & 1.0 & 1.7 \\
3 & 1.3 & 0.7 & 8.1 & 3.4 \\
4 & 1.4 & 1.5 & 1.7 & 1.3 \\
5 & 4.1 & 2.3 & 2.6 & 6.4 \\
6 & 2.9 & 1.5 & 1.5 & 1.2 \\
7 & 1.2 & 0.9 & 1.2 & 2.4 \\
8 & 2.4 & 2.5 & 5.3 & 5.4 \\
9 & 3.1 & 2.5 & 2.4 & 2.4 \\
10 & 2.0 & 2.5 & 2.1 & 2.7 \\
11 & 4.5 & 2.8 & 2.1 & 2.4 \\
Mean & 2.3 & 1.8 & 2.6 & 2.8 \\
& $(\mathrm{SD} \pm 1.2)^{\mathrm{a}}$ & $(\mathrm{SD} \pm 0.8)^{\mathrm{a}}$ & $(\mathrm{SD} \pm 2.2)^{\mathrm{a}}$ & $(\mathrm{SD} \pm 1.7)^{\mathrm{a}}$ \\
\hline
\end{tabular}

${ }^{\mathrm{a}}$ No significant difference $(p>0.05)$.

Only one bolus per viscosity was used, therefore no data on intrasubject differences could be provided. If multiple boluses per viscosity had been used in this study, the radiation exposure for our volunteers would have been much higher. Because none of the volunteers had swallowing disorders and all were used to swallowing tap water and yogurt, any adaptation in swallowing during the study to the circumstances during data collection or intrasubject differences can be considered negligible.

Raut et al. [13] examined with manometry the influence of increased viscosity on pharyngeal pressures. It was concluded that an increased bolus viscosity led to increased amplitude of the bolus wave and clearing contractions within the pharynx. The increase in contraction of the pharyngeal muscles supports our findings of equivalent percentages of residue for the different viscosities.

Hamlet et al. [11] reported on the differences in bolus transit times and oropharyngeal residues in healthy volunteers. In that study 20 healthy volunteers (age $=39-65$ years) swallowed tap water and a thickened substance labeled with ${ }^{99 \mathrm{~m}} \mathrm{Tc}$. The mean pharyngeal residue was found to be $4.0 \%$ (standard error $[\mathrm{SE}]=0.5)$ for tap water and $10.5 \%$ $(\mathrm{SE}=0.1)$ for a substance with a viscosity of 1070 $\mathrm{mPa}$ s.

Differences in the Hamlet et al. study and our study might be explained by temperature influences and different approaches to measure viscosity. In the Hamlet study [11] the bolus temperature was set at $25^{\circ} \mathrm{C}$, whereas in our study syringes were stored in a chilled room. Therefore, temperature is not consid- ered a confounding factor in our study. Also, in the Hamlet study [11] a Brookfield Viscometer type RVT was used, which is more suitable for measuring the viscosity of low-viscosity products like milk. In our study we used a Brookfield Viscometer type LVT, which is more suitable for high-viscosity substances, making an adequate comparison between measured viscosities of the two studies not possible.

Literature suggests that body positioning, bolus temperature, and taste affect swallowing $[3,4,14]$. In our study the volunteers had to rotate their heads to the right side to obtain adequate measurements, following the protocol used in the Hamlet study [11]. This might have reduced the amount of pharyngeal residue because the bolus passes the pharynx mainly on one side and the contact area between bolus and pharyngeal wall is decreased. It is known that in some dysphagic patients (i.e., patients with unilateral pharyngeal weaknesses) rotating the head decreases the amount of residue, but the effects of head rotation on healthy individuals has not been investigated. Looking at the wide distribution of percentages of residues in volunteers (Table 1) and the slightly lower percentage (2.3\%) found in our study compared with that of Shaw [12] $(2.5 \%)$, where measurements were made in a right lateral position, one might conclude that the influence of head rotation in our study cannot be seen as a confounding factor on the outcome.t

\section{Conclusion}

In 11 healthy volunteers, mean pharyngeal residue after swallowing tap water was $2.3 \%(\mathrm{SD}=1.2)$ of the initial volume in the oral cavity. The percentage of pharyngeal residue after swallowing $0.5 \%$ xanthan solution was $1.8 \%(\mathrm{SD}=0.8)$, after swallowing $0.75 \%$ xanthan solution the residue was $2.6 \%$ $(\mathrm{SD}=2.2)$, and after swallowing $1.00 \%$ xanthan solution the residue was $2.8 \%(\mathrm{SD}=1.7)$. Our data do not show a significant increase in pharyngeal residue in healthy volunteers as a result of an increasing viscosity of viscous solutions, ranging from tap water to full-fat yogurt. Although some trends may be seen, (confounding) factors other than viscosity may play a role in the build up of pharyngeal residue.

Acknowledgments. The authors thank Mrs. L. Hooft, the Department of Clinical Epidemiology and Biostatistics, Academic Medical Center, University of Amsterdam, for additional statistical analyses. 


\section{References}

1. Perlman AL, Booth BM, Grayhack JP: Videofluoroscopic predictors of aspiration in patients with oropharyngeal dysphagia. Dysphagia 9(2):90-95, 1994

2. Dejaeger E, Pelemans W, Ponette E, Joosten E: Mechanisms involved in postdeglutition retention in the elderly. Dysphagia 12(2):63-67, 1997

3. Bisch EM, Logemann JA, Rademaker AW, Kahrilas PJ, Lazarus CL: Pharyngeal effects of bolus volume, viscosity, and temperature in patients with dysphagia resulting from neurologic impairment and in normal subjects. $J$ Speech Hear Res 37(5):1041-1059, 1994

4. Logemann JA, Pauloski BR, Colangelo L, Lazarus C, Fujiu M, Kahrilas PJ: Effects of a sour bolus on oropharyngeal swallowing measures in patients with neurogenic dysphagia. J Speech Hear Res 38(3):556-563, 1995

5. Stoeckli SJ, Huisman TA, Seifert B, Martin-Harris BJ: Interrater reliability of videofluoroscopic swallow evaluation. Dysphagia 18(1):53-77, 2003

6. Tatsch K, Schroettle W, Kirsch CM: Multiple swallow test for the quantitative and qualitative evaluation of esophageal motility disorders. J Nucl Med 32(7):1365-1370, 1991

7. Bennink R, Peeters M, Van den Maegdenbergh V, Geypens B, Rutgeerts P, De Roo M, Mortelmans L: Comparison of total and compartmental gastric emptying and antral motility between healthy men and women. Eur J Nucl Med 25(9):12931299, 1998
8. Vandenplas Y, Derde MP, Piepsz A: Evaluation of reflux episodes during simultaneous esophageal $\mathrm{pH}$ monitoring and gastroesophageal reflux scintigraphy in children. $J$ Pediatr Gastroenterol Nutr 14(3):256-260, 1992

9. Argon M, Secil Y, Duygun U, Aydogdu I, Kocacelebi K, Ozkilic H, Ertekin C: The value of scintigraphy in the evaluation of oropharyngeal dysphagia. Eur J Nucl Med Mol Imaging 31(1):94-98, 2004

10. Cook IJ, Weltman MD, Wallace K, Shaw DW, McKay E, Smart RC, Butler SP: Influence of aging on oral-pharyngeal bolus transit and clearance during swallowing: scintigraphic study. Am J Physiol 266(6 Pt 1):972-977, 1994

11. Hamlet S, Choi J, Zormeier M, Shamsa F, Stachler R, Muz J, Jones L: Normal adult swallowing of liquid and viscous material: scintigraphic data on bolus transit and oropharyngeal residues. Dysphagia 11(1):41-47, 1996

12. Shaw DW, Williams RB, Cook IJ, Wallace KL, Weltman MD, Collins PJ, McKay E, Smart R, Simula ME: Oropharyngeal scintigraphy: a reliable technique for the quantitative evaluation of oral-pharyngeal swallowing. Dysphagia 19(1):36-42, 2004

13. Raut VV, McKee GJ, Johnston BT: Effect of bolus consistency on swallowing - does altering consistency help? Eur Arch Otorhinolaryngol 258(1):49-53, 2001

14. Logemann JA, Rademaker AW, Pauloski BR, Kahrilas PJ: Effects of postural change on aspiration in head and neck surgical patients. Otolaryngol Head Neck Surg 110(2):222227, 1994 